\section{KETOACIDOSIS AND FLUIDS}

The debate around fluid resuscitation and maintenance in DKA has been smouldering for years, the recent, large PECARN FLUID trial providing some guidance, but, not drawing a line under all the issues

In the light of the study, revisiting the arguments is useful and a group of three papers re-open the discussion. The catalyst on this occasion has been the publication of new British Society of Paediatric Endocrinology (BSPED) guidance, recommendations which leave ultimate decision making to the individual clinician but in broad terms suggest an initial resuscitation bolus (of $10 \mathrm{~mL} / \mathrm{kg}$ ) to all children. Our first correspondent, John Lillie on behalf of the South Thames Retrieval Service whose policy has been restrictive since 2008 after three deaths from DKA associated cerebral oedema argues that degree of dehydration (an agreed moot point by all parties) is all too easily overestimated particularly when capillary refill time (prolonged by hypocapnoea inherent to ketosis) is used to make the assessment. Neil Wright on behalf of BPSED argues that once initial resuscitation is completed there is little difference philosophically between the two approaches

The physiology, science and moot points are weighed up in Robert Tasker's editorial in which one bystander in recent debate, the rate of insulin infusion is also revisited, a lower exposure causing less rapid shifts in osmotic pressure and (theoretically) less risk of cerebral oedema. Here we come full circle in that the number of children developing this complication is so low that even a trial as large as FLUID is potentially underpowered. See pages 1019, 1020 and 917

\section{PERINATAL ENCEPHALOPATHY}

The dangers of over-diagnosis of a vague entity are highlighted in Mustayev's systematic review. The term perinatal encephalopathy (PE) (sometimes also called the 'syndrome of intracranial

Department of Women's and Children's Health, International Maternal and Child Health (IMCH), Uppsala University, Uppsala, Sweden; Department of Paediatrics, Länssjukhuset Gävle-Sandviken, Gävle, Sweden; Department of Child Health, Aga Khan University, Karachi, Pakistan

Correspondence to Dr Nick Brown, Department of Women's and Children's Health, International Maternal and Child Health (IMCH), Uppsala University, Uppsala 75237, Sweden; nickjwbrown@gmail.com hypertension') was coined by a Russian paediatrician Iurii Iakunin in the 1970s referring to a range of signs and symptoms thought to be attributable to a perinatal insult, mediated by a rise in intracranial pressure. The notion was admirable, but the group of disorders inevitably heterogenous. As the term became more widely used in Eastern European countries, it was sometimes applied to infants and children with transient signs and no discernable pathology. The nomenclature was (paradoxically) reinforced by the lack of a unifying diagnostic test; the label being at the discretion of the paediatrician or paediatric neuropathologist, to which many of these infants were referred. Diagnoses result in treatments and wide range of agents had been used on occasions: anticonvulsants, mineral and metabolic supplements, diuretics, cattle-derived neuropeptides, vasoactive agents, psychostimulants, and physical therapies. The issue of the Perinatal Encephalopathy Syndrome has long been on the radar of the WHO, and was the subject of a meeting in St Petersburg in 2007, at which many positive signs of reform were seen. This review shows further change, but some areas of continuing concern related to the diagnosis which still appears to be applied in some areas. These potential harms are both direct and indirect and include the failure to diagnose other disorders; unnecessary follow-up appointments and diagnostic procedures; the development of the vulnerable child syndrome; and even deferral of vaccinations. See page 921

\section{AFTER SUDDEN INFANT DEATH}

SUDI is a rare event and a second death in a subsequent child extremely unusual, but to date there has been little data to quantify the recurrence risk and counsel parents. Garstang's analysis of the Care of the Next Infant database from 2000 to 2015 provides some answers. Over this period, 6608 live-born infants were registered: 171 were first-born infants to mothers whose male partners had previously had an unexplained infant death. 29 unexpected infant deaths following the index death occurred in 26 families, 23 with 2 deaths and 3 with three deaths. The second SUDI rate was estimated as 3.93 per 1000 live births and the third as 115 per 1000 live births. The findings should not, though, engender complacency as there have in the past been convictions for homicide. The risk of repeat SUDI in a family is still 10 times that of the general population, a reflection of inherent genetic risks as well as environmental factors such as maternal smoking and unsafe sleeping. CONI cannot address intrinsic risk factors, but these are very vulnerable families who need comprehensive care and support packages to help them understand safe sleeping, address mental health problems and enhance their parenting capacity. See page 945

\section{EMERGENCY STEROIDS AND ASTHMA PROPHYLAXIS}

In a neat and salutary reminder of the reason some children reach the stage of requiring rescue oral corticosteroids (OCS) at routine clinic appointments, Willson reviews experience from a quarternary respiratory department with respect to adherence prescribed prophylaxis. In the series 25 children received 32 courses of OCS. For those episodes with full data, uptake of prescriptions for inhaled corticosteroid prophylaxis, the median uptake over the previous 6 months was only $33 \%$ and in only $29 \%$ episodes was uptake $\geq 75 \%$ of that prescribed So, rather than just prescribe the emergency course and ascribe it to bad luck or bad asthma... maybe check on adherence. This and related themes are explored in Ian Sinha's Viewpoint exploration of the national respiratory audit database. See pages 993 and 910

\section{MONITORING INFLAMMATORY BOWEL DISEASE}

Equally pragmatic is the issue with calprotectin stability described by Haisma. Stool calprotectin is pivotal in the diagnosis, monitoring of and to treatment modifications in IBD. Often a sample will be taken in the home and dropped off at the lab or sent by post having spent time at room temperature in the interim rather than the recommended $4 \mathrm{C}$. The fall in levels is so great (35\% and $46 \%$ in extraction buffer) that disease activity will inevitably be underestimated and treatment not increased appropriately. So, before reducing immune modulating treatment immediately, check how the sample travelled before analysis and, if in any doubt, recheck making any changes. See page 996

\section{ORCID iD}

Nick Brown http://orcid.org/0000-0003-1789-0436 